\title{
INTEGRAL LOGARITHMIC MEANS FOR REGULAR FUNCTIONS
}

\section{N. LINDEN}

For a function $f$, regular in the unit disc, integral logarithmic means are defined by the formulae

$$
M_{p}(r, f)=\left\{\frac{1}{2 \pi} \int_{0}^{2 \pi}|\log | f\left(r e^{i \theta}\right)||^{p} d \theta\right\}^{1 / p} \quad(0<r<1)
$$

for $0<p<\infty$. These are related to

$$
M_{\infty}(r, f)=\sup _{|z|=r}|\log | f(z)|| \quad(0<r<1)
$$

when the latter increases sufficiently rapidly. Thus when $\lambda_{\infty}(f) \geq 1$ the orders

$$
\lambda_{p}(f)=\limsup _{r \rightarrow 1} \frac{\log M_{p}(r, f)}{\log 1 /(1-r)}
$$

are continuous at infinity in the sense that

$$
\lim _{p \rightarrow \infty} \lambda_{p}(f)=\lambda_{\infty}(f),
$$

a property which does not generally hold when $\lambda_{\infty}(f)<1$. It transpires that in the extreme cases $\lambda_{\infty}(f)=\lambda_{1}(f)+1$, and $\lambda_{\infty}(f)=$ $\lambda_{1}(f) \geq 1, \lambda_{p}(f)$ is uniquely determined for $1<p<\infty$.

1. Introduction. For a given function $f$, regular in the unit disc $D(0,1)=\{z:|z|<1\}$ let

$$
\begin{aligned}
& M_{p}(r, f)=\left\{\frac{1}{2 \pi} \int_{0}^{2 \pi}|\log | f\left(r e^{i \theta}\right)||^{p} d \theta\right\}^{1 / p} \quad(0<p<\infty) \\
& M_{\infty}(r, f)=\sup _{|z|=r} \log |f(z)|
\end{aligned}
$$

for $0 \leq r<1$. We consider the asymptotic values of these quantities as $r \rightarrow 1$ in terms of the orders

$$
\lambda_{p}(f)=\limsup _{r \rightarrow 1} \frac{\log M_{p}(r, f)}{\log (1 /(1-r))} .
$$

Note that $\lambda_{1}(f)$ is equal to the Nevanlinna order of $f$, and $\lambda_{\infty}(f)$ is the maximum modulus order of $f$, related by the classical inequalities

$$
\lambda_{1}(f) \leq \lambda_{\infty}(f) \leq \lambda_{1}(f)+1,
$$


which are readily obtained from the Poisson-Jensen formula [6, p. 205].

Certain properties of $\lambda_{p}(f)$ follow immediately from the Hölder inequalities (see [4, pp. 9 and 15] for the corresponding properties of $\left.M_{p}(r, f)\right)$; for example

(A) $\lambda_{p}(f)$ is an increasing function of $p(0<p<\infty)$,

(B) $p \lambda_{p}(f)$ is convex on the interval $(0, \infty)$.

In contrast, $\lambda_{\infty}(f)$ does not generally fit naturally into this context. For elementary calculations show that if $0<\alpha<1$ then

$$
F(z)=\exp \left\{(1+z)^{-\alpha}-(1-z)^{-1}\right\} \quad(|z|<1)
$$

satisfies $\lambda_{\infty}(F)=\alpha$, while $\lambda_{p}(F)=1-1 / p$ for $p>1$. Nevertheless, we will show that

$$
\lambda_{\infty}(f)=\lim _{p \rightarrow \infty} \lambda_{p}(f)
$$

provided that $\lambda_{\infty}(f)$ is sufficiently large by proving the following result.

THEOREM 1. If $f$ is regular in $D(0,1)$ and $\lambda_{\infty}(f) \geq 1$, then

$$
\begin{gathered}
\lambda_{p}(f) \leq \lambda_{\infty}(f), \\
\lambda_{\infty}(f) \leq \lambda_{p}(f)+1 / p
\end{gathered}
$$

for $0<p \leq \infty$. Thus (1.2) holds when $\lambda_{\infty}(f) \geq 1$.

The following corollary is deduced readily from Theorem 1 in $\S 4$.

Corollary 1. If $f$ is regular in $D(0,1)$ and $\lambda_{\infty}(f) \geq 1$, then

(i) $p\left(\lambda_{\infty}(f)-\lambda_{p}(f)\right)$ is an increasing function of $p$ on $(0, \infty)$, with range contained in $[0,1]$,

(ii) $\lambda_{p}(f)+1 / p$ is a decreasing function of $p$ on $(0, \infty]$.

When $p=1$, the inequalities (1.3) and (1.4) are equivalent to (1.1); in the case $p=2$ they have been obtained by Sons [5]. As far as one extreme case of the inequalities (1.1) is concerned, it is readily observed that condition (A) shows that the equality $\lambda_{\infty}(f)=\lambda_{1}(f)$ implies that $\lambda_{p}(f)=\lambda_{1}(f)$ for $p \geq 1$. In the other extreme case represented by

$$
\lambda_{\infty}(f)=\lambda_{1}(f)+1
$$

$\lambda_{p}(f)$ is also completely determined when $p \geq 1$, since Corollary 1(ii) implies

$$
\lambda_{\infty}(f) \leq \lambda_{p}(f)+1 / p \leq \lambda_{1}(f)+1 \quad(1 \leq p \leq \infty) .
$$

Thus we obtain a second corollary. 
CoRollary 2. If $f$ is regular in $D(0,1)$ and $\lambda_{\infty}(f)=\lambda_{1}(f)+1$, then

$$
\lambda_{p}(f)=\lambda_{\infty}(f)-1 / p \quad(p \geq 1) .
$$

2. Preliminaries for the proof of Theorem 1. In this section, we assemble some background material needed for the proof of Theorem 1. We put $\lambda_{\infty}(f)=\lambda$ and, when $\lambda$ is finite, let $\mu$ be the integer satisfying

$$
\lambda<\mu \leq \lambda+1 \text {. }
$$

Then for each given positive number $\varepsilon$ we have

$$
\log \left|f\left(r e^{i \theta}\right)\right|<(1-r)^{-\lambda-\varepsilon} \quad\left(r_{0} \leq r<1,0 \leq \theta<2 \pi\right)
$$

for some $r_{0}$ in $(0,1)$.

We later seek lower bounds for $\log \left|f\left(\mathrm{re}^{i \theta}\right)\right|$ by considering a factorisation based on the zero sequence $\left\{a_{m}\right\}$ of $f$ in $D(0,1) \backslash\{0\}$, each zero being counted according to multiplicity. Let

$$
b\left(z, a_{m}, \mu\right)=\left(1-\frac{1-\left|a_{m}\right|^{2}}{1-z \bar{a}_{m}}\right) \exp \sum_{j=1}^{\mu} \frac{1}{j}\left(\frac{1-\left|a_{m}\right|^{2}}{1-z \bar{a}_{m}}\right)^{j} .
$$

This leads to the factorisation

$$
f(z)=g(z) z^{s} B\left(z,\left\{a_{m}\right\}, \mu\right),
$$

where

$$
B(z)=B\left(z,\left\{a_{m}\right\}, \mu\right)=\prod_{m} b\left(z, a_{m}, \mu\right),
$$

$s$ is a nonnegative integer, and $g(z)$ is regular and nonzero in $D(0,1)$.

The result (1.4), is readily obtained for $g(z)$ by a simple application of a known theorem [1, p. 84]. We need to show that it also applies to the factor $B(z)$. We require some known results, the first being a theorem of Tsuji [6, p. 224].

TheOREM A. For the canonical product $B(z)$ defined by (2.3), and positive $\varepsilon$ we have

$$
\log |B(z)| \leq K \sum_{m}\left|\frac{1-\left|a_{m}\right|^{2}}{1-z \bar{a}_{m}}\right|^{\mu+1+\varepsilon} \quad\left(\frac{1}{2} \leq|z|<1\right),
$$

and, if $C_{m}$ denotes the disc $D\left(a_{m},\left(1-\left|a_{m}\right|^{2}\right)^{\mu+4}\right)$ then

$$
\log |B(z)| \geq K \log (1-|z|) \sum_{m}\left|\frac{1-\left|a_{m}\right|^{2}}{1-z \bar{a}_{m}}\right|^{\mu+1+\varepsilon}
$$

when $\frac{1}{2} \leq|z|<1, z \notin \bigcup_{m} C_{m}$. 
The constant $K$ in (2.4) and (2.5) depends on $\varepsilon, \mu$ and $\left\{a_{m}\right\}$, or on $\varepsilon$ and $f$ if we regard $B(z)$ as defined by (2.2). As here, we will subsequently use $K$ to denote a positive constant, not necessarily the same at each occurrence, but depending on parameters which will normally be stipulated as appropriate. The symbol $r_{0}$ will be used similarly, but always restricted to the interval $(0,1)$.

We require some information regarding the zero distribution of $f$ when $\lambda_{\infty}(f)=\lambda \geq 1$. Let the disc $D(0,1)$ be covered by sets of the form

$S(q, k)=\left\{z: 1-2^{-q} \leq|z|<1-2^{-q-1}, \pi k 2^{-q} \leq \arg z<\pi(k+1) 2^{-q}\right\}$

for integers $q$ and $k$ satisfying

$$
q=0,1,2, \ldots, \quad-2^{q} \leq k<2^{q}-1 .
$$

For the given function $f$ let $N(q, k, f)$ denote the number of zeros of $f$ in $S(q, k)$. Then for any positive $\varepsilon$ there is a number $q_{0}$, such that

$$
N(q, k, f)<2^{(\lambda+\varepsilon) q} \quad\left(q \geq q_{0}\right),
$$

for all relevant $k$ in (2.6) [3, p. 21]. This inequality gives rise to a bound to the sums occurring in (2.4) and (2.5), as estimated in [3, pp. 23-25].

THEOREM B. Let $f$ be regular in $D(0,1)$ with factorisation (2.2). Then for each positive $\varepsilon$, and $\alpha>\lambda=\lambda_{\infty}(f) \geq 1$, we have

$$
\sum_{m}\left|\frac{1-\left|a_{m}\right|^{2}}{1-z \bar{a}_{m}}\right|^{\alpha+1}<K(1-|z|)^{-\lambda-\varepsilon} \quad\left(r_{0} \leq|z|<1\right)
$$

for some $r_{0}$ in $(0,1)$.

As a final preliminary to the proof of (1.3) of Theorem 1, we estimate $M_{p}(r, f)$ according to the following lemma.

LEMMA 1. Let $f$ be regular in $D(0,1)$ and $\lambda_{\infty}(f)=\lambda \geq 1$. Then, if $\varepsilon>0$ and $1 \leq p<\infty$ we have

$$
\int_{0}^{2 \pi}|\log | B\left(r e^{i \theta},\left\{a_{m}\right\}, \mu\right)||^{p} d \theta<K(1-r)^{-p(\lambda+\varepsilon)}
$$

for some constant $K$ and $0 \leq r<1$. 
We deal with the integral in (2.8) by covering the range of integration by $[\pi /(1-r)]+1$ intervals of the form $[\tau+r-1, \tau+1-r]$ for $\tau=2 k(1-r)$ and $k=0,1,2, \ldots,[\pi /(1-r)]$, showing that

$$
\int_{\tau+r-1}^{\tau+1-r}|\log | B\left(r e^{i \theta}\right)||^{p} d \theta<K(1-r)^{1-p(\lambda+\varepsilon)}
$$

for each $\tau$. The method of proof indicates that the constant $K$ need not depend on $\tau$. However, for convenience and without loss of generality, we suppose that $\tau=0$ in the following proof. Thence we obtain (2.8), as stated.

Without loss of generality, we assume

$$
\frac{1}{2} \leq r<1, \quad \frac{3}{4} \leq\left|a_{m}\right|<1,
$$

since the contribution to the integral (2.8), due to any zeros not satisfying this latter inequality is clearly bounded. For given $r$, let $E$ denote the set of integers $m$ for which the exceptional discs $C_{m}$ of Theorem A intersect $\gamma_{r}=\left\{z: z=r e^{i \theta}, r-1 \leq \theta \leq 1-r\right\}$. By application of (2.7), we have

$$
\#(E)<K(1-r)^{-\lambda-\varepsilon},
$$

where $\#(E)$ denotes the number of elements in the set $E$. We consider the factorisation $B=B_{1} B_{2} B_{3}$, where

$$
\begin{aligned}
& B_{1}(z)=\prod_{m \notin E} b\left(z, a_{m}, \mu\right), \\
& B_{2}(z)=\prod_{m \in E} \exp \sum_{j=1}^{\mu} \frac{1}{j}\left(\frac{1-\left|a_{m}\right|^{2}}{1-z \bar{a}_{m}}\right)^{j}, \\
& B_{3}(z)=\prod_{m \in E} 1-\frac{1-\left|a_{m}\right|^{2}}{1-z \bar{a}_{m}}=\prod_{m \in E} \frac{\bar{a}_{m}\left(a_{m}-z\right)}{1-z \bar{a}_{m}} .
\end{aligned}
$$

First we note that for any positive number $\varepsilon$, Theorems A and B give

$$
\begin{aligned}
\int_{r-1}^{1-r}|\log | B_{1}\left(r e^{i \theta}\right)||^{p} d \theta & <K(1-r)^{1-p(\lambda+\varepsilon / 2)} \log \left(\frac{1}{1-r}\right)^{p} \\
& <K(1-r)^{1-p(\lambda+\varepsilon)}
\end{aligned}
$$

where the constants $K$ in (2.12) can be chosen to depend only on $\varepsilon, \mu, p$, and the whole sequence $\left\{a_{m}\right\}$.

Next, the inequality

$$
\left|1-z \bar{a}_{m}\right|>\frac{1}{2}\left(1-\left|a_{m}\right|^{2}\right)
$$


yields

$$
|\log | B_{2}(z)||<\sum_{m \in E} \frac{1}{j}\left|\frac{1-\left|a_{m}\right|^{2}}{1-z \bar{a}_{m}}\right|^{j} \leq K \#(E) .
$$

Hence (2.11) implies

$$
\int_{r-1}^{1-r}|\log | B_{2}\left(r e^{i \theta}\right)||^{p} d \theta<K(1-r)^{1-p(\lambda+\varepsilon)} .
$$

It remains to consider $B_{3}$.

Given $z=\mathrm{re}^{i \theta}$ in $D(0,1)$ we have

$$
1 \geq\left|B_{3}(z)\right|^{2}=\prod_{m \in E} r_{m}^{2}\left\{1+\frac{\left(1-r^{2}\right)\left(1-r_{m}^{2}\right)}{\left|z-a_{m}\right|^{2}}\right\}^{-1}
$$

where $a_{m}=r_{m} e^{i \theta_{m}}$. For each $m$ in $E$ we can find $w$ with $|w|=r$ such that

$$
\left|w-a_{m}\right| \leq\left(1-\left|a_{m}\right|^{2}\right)^{\mu+4} \leq\left(\frac{7}{16}\right)^{3}\left(1-r_{m}^{2}\right)<\frac{1}{8}\left(1-r_{m}^{2}\right) .
$$

Thus

$$
1-r_{m}^{2}<2\left(1-r_{m}\right) \leq 2\left(1-r+\left|w-a_{m}\right|\right),
$$

from which we obtain

$$
1-r_{m}^{2}<\frac{8}{3}(1-r)<\frac{8}{3}\left(1-r^{2}\right) .
$$

Since

$$
\left|z-a_{m}\right|^{2} \geq 4 r r_{m} \sin ^{2} \frac{1}{2}\left(\theta-\theta_{m}\right) \geq \frac{3}{2} \sin ^{2} \frac{1}{2}\left(\theta-\theta_{m}\right),
$$

and in (2.14),

$$
\sum_{m \in E} \log \left(\frac{1}{r_{m}}\right)<\#(E) \log \left(\frac{4}{3}\right),
$$

Minkowski's inequality yields

$$
\begin{aligned}
\int_{r-1}^{1-r} & |\log | B_{3}\left(r e^{i \theta}\right)||^{p} d \theta \\
& <K \#(E)^{p}(1-r)+K \int_{r-1}^{1-r}\left(\sum_{m \in E} \log 1+\frac{16\left(1-r^{2}\right)^{2}}{9 \sin ^{2} \frac{1}{2}\left(\theta-\theta_{m}\right)}\right)^{p} d \theta \\
& <K \#(E)^{p}(1-r)+K \#(E)^{p} \int_{r-1}^{1-r}\left(\log \left(1+\frac{16\left(1-r^{2}\right)^{2}}{9 \sin ^{2} \frac{1}{2} t}\right)\right)^{p} d t \\
& <K \#(E)^{p}(1-r) .
\end{aligned}
$$


The inequality (2.9) with $\tau=0$ now follows from (2.11), (2.12), (2.13) and this last inequality, so that Lemma 1 is proved.

3. The Proof of Theorem 1. We begin the proof of Theorem 1 by using the results of the last section to verify (1.3). The property (A) shows that, without any loss of generality, we may assume $p>1$.

Let $\varepsilon$ be a given number in the interval $(0, \mu-\lambda)$. Then in applying Tsuji's Theorem A, we note

$$
\sum_{r<\left|a_{m}\right|<1}\left(1-\left|a_{m}\right|^{2}\right)^{\mu+4}<\left(1-r^{2}\right)^{2} \sum_{r<\left|a_{m}\right|<1}\left(1-\left|a_{m}\right|^{2}\right)^{\mu+2},
$$

where this latter sum converges. Therefore, there is an integer $q_{0}$ such that each interval $\left[1-2^{-q}, 1-2^{-q-1}\right)$ contains a number $R_{q}$ for which the circle $\left\{z:|z|=R_{q}\right\}$ does not intersect any of the exceptional discs of Theorem A when $q \geq q_{0}$. An application of Theorem A implies

$$
\begin{array}{r}
|\log | B\left(z,\left\{a_{m}\right\}, \mu\right)||<K(1-|z|)^{-\lambda-\varepsilon} \log (1 /(1-|z|)) \\
\left(|z|=R_{q}, q \geq q_{0}\right) .
\end{array}
$$

By using the factorisation (2.2), we now have

$$
\begin{aligned}
\log |g(z)| & \leq \log |f(z)|+|\log | B\left(z,\left\{a_{m}\right\}, \mu\right)||-s \log |z| \\
& \leq K(1-|z|)^{-\lambda-\varepsilon} \log (1 /(1-|z|)) \quad\left(|z|=R_{q}, q \geq q_{0}\right) .
\end{aligned}
$$

Hence, for any $r$ in $\left[1-2^{-q}, 1-2^{-q-1}\right)$, the maximum modulus principle implies

$$
\begin{aligned}
M_{\infty}(r, g) & \leq M\left(R_{q+1}, g\right) \leq K(q+1) 2^{(q+1)(\lambda+\varepsilon)} \\
& <K(1-r)^{-\lambda-\varepsilon} \log (1 /(1-r))
\end{aligned}
$$

when $q \geq q_{0}$. Since $\lambda \geq 1$, and $g$ has no zeros in $D(0,1)$, it follows [2] that

$$
\begin{array}{r}
|\log | g(z)|| \leq K(1-|z|)^{-\lambda-\varepsilon} \log (1 /(1-|z|)) \\
\left(1-2^{-q_{0}} \leq|z|<1\right) .
\end{array}
$$

The inequality (3.2) leads to

$$
M_{p}(r, g) \leq K(1-r)^{-\lambda-\varepsilon} \log (1 /(1-r)),
$$

from which the Minkowski inequalities and Lemma 1 yield

$$
\begin{aligned}
M_{p}(r, f) & \leq M_{p}(r, g)+M_{p}(r, B) \\
& \leq K(1-r)^{-\lambda-\varepsilon} \log (1 /(1-r))
\end{aligned}
$$


when $r$ is sufficiently close to 1 . We now have $\lambda_{p}(f) \leq \lambda+\varepsilon$ for all positive $\varepsilon$, so that

$$
\lambda_{p}(f) \leq \lambda=\lambda_{\infty}(f)
$$

The inequality (1.3) has been proved.

The proof of (1.4) when $p>1$ is obtained by applying the method of proof of Theorem 5.9 [1, p. 84]. The Poisson-Jensen formula, together with Hölder's inequality, yields

$$
\begin{aligned}
\log \left|f\left(r e^{i \theta}\right)\right| & \leq \frac{1}{2 \pi} \int_{0}^{2 \pi}|\log | f\left(R e^{i \phi}\right)|| P(R, r, \theta-\phi) d \phi \\
& \leq M_{p}(r, f)\left(\frac{1}{2 \pi} \int_{0}^{2 \pi} P(R, r, \theta-\phi)^{p / p-1} d \phi\right)^{(p-1) / p},
\end{aligned}
$$

for $0<r<R<1,0 \leq \theta<2 \pi$. We put $R=\frac{1}{2}(1+r)$, and use a standard estimate [1, p. 84] for the Poisson kernel to obtain

$$
M_{\infty}(r, f) \leq K M_{p}(r, f)(1-r)^{-1 / p} .
$$

The inequality (1.4) follows for $1<p<\infty$, and so does (1.2).

We have already noted that (1.1) implies (1.4) when $p=1$, so it remains to consider $0<p<1$. The property (B) shows that

$$
p\left(\lambda_{s}(f)-\lambda_{p}(f)\right) \leq q\left(\frac{s-p}{s-q}\right)\left(\lambda_{s}(f)-\lambda_{q}(f)\right) \quad(0<p<q<s),
$$

with limiting form

$$
p\left(\lambda_{\infty}(f)-\lambda_{p}(f)\right) \leq q\left(\lambda_{\infty}(f)-\lambda_{q}(f)\right) \quad(0<p<q),
$$

obtained from (1.2). But we have already seen that the right-hand side of this latter inequality has upper bound 1 when $q>1$. Hence $0<p<1<q$ implies

$$
p\left(\lambda_{\infty}(f)-\lambda_{p}(f)\right) \leq 1
$$

for $0<p<1$, and (1.4) follows for all positive $p$.

4. The proof of Corollary 1. Corollary 1 follows readily from the proof of Theorem 1 . The inequality (3.5) shows that $p\left(\lambda_{\infty}(f)-\lambda_{p}(f)\right)$ is increasing on $(0, \infty)$, and (1.3) and (1.4) show that the range of this function is included in $[0,1]$. The inequalities (3.5) and (1.4) also 
imply

$$
\begin{aligned}
p \lambda_{p}(f) & \geq q \lambda_{q}(f)-(q-p) \lambda_{\infty}(f) \\
& \geq q \lambda_{q}(f)-(q-p)\left(\lambda_{q}(f)+1 / q\right)=p \lambda_{q}(f)-1+p / q .
\end{aligned}
$$

Corollary 1(ii) follows immediately for $0<p<\infty$, and for $p=\infty$ by taking limits.

\section{REFERENCES}

[1] P. Duren, Theory of $H^{p}$ Spaces, Academic Press, 1970.

[2] C. N. Linden, Functions regular in the unit circle, Proc. Camb. Phil. Soc., (1956), 49-60.

[3] $\frac{}{19-30 .}$, The representation of regular functions, J. London Math. Soc., 39 (1964),

[4] J. E. Littlewood, Theory of Functions, Oxford University Press, 1944.

[5] L. R. Sons, Zero distribution of functions with slow or moderate growth in the unit disc, Pacific J. Math., 99 (1982), 473-481.

[6] M. Tsuji, Potential Theory, Tokyo, 1959.

Received November 23, 1987.

University College of Swansea

SINGLETON PARK

SWANSEA SA2 8PP WALES 
